Aus der chirurgischen Universitätsklinik zu Göttingen

(Direktor: Geh. Medizinalrat Prof. Dr. Braun).

\title{
Zur Frage der chirurgischen Behandlung von Typhusbazillenträgern.
}

Von Dr. Albert Fromme, Privatdozent und Assistenzarzt der Klinik.

Daß Bezichungen zwischen Typhus abdominalis und der Pathologie des Gallensystems bestehen, ist schon schr lange bekannt und schon zu einer Zeit, als der Erreger des Typhus noch nicht entdeckt war, haben sich zahlreiche Autoren mit dem $\mathrm{Zu}$ sammenhang zwischen diesen Erkrankungen beschäftigt. Der erste, dem der Nachweis von Typhusbazillen in der Gallenblase gelang, war F üt tere ${ }^{1}$ ). Seit dieser Zeit konnte der Nachweis schr häufig erbracht werden. Aber obwohl eine große Reihe Arbeiten in dieser Frage erschienen sind, herrscht über den Modus der Infektion noch keine Ubereinstimmung und wir kennen noch nicht die Gründe für die unter Umständen zurückbleibenden Krankheiten des Gallensystems. In zusammenhängenden Referaten wurden diese Fragen im Jahre I907 in der Deutschen Pathologischen Gesellschaft ${ }^{2}$ ) behandelt und zwar vom Standpunkt des pathologischen Anatomen ${ }^{3}$ ), des Klinikers ${ }^{4}$ ) und des Bakteriologen ${ }^{5}$.

1) A n ton und $F$ üt $t$ erer, Cntersuchungen über Typhus abdominalis. Münchener medizinische Wochenschrift I888. Nr. 19.

2) Verhandungen der Deutschen Pathologischen Gesellschaft 1907, Nr. I1, S. 143 folgende (hier findet sich cin vollständiges Literaturverzeichnis, auf das bezüglich der älteren Literatur verwiesen wird).

3) Chiari, '́ber 'Typhus abdominalis und Paratyphus in ihren Beziehungen zu den Gallenwegen.

4) $\mathrm{H}$ irsch, Erkrankungen der Leber und Gallenwege bei Typhus.

5) Forster, ther die Beziehungen des Typhus und Paratyphus zu den Gallenwegen. 
Für die Infektion der Gallenblase kommen beim Typhuskranken zwei Wege in Betracht. Erstens aszendierend vom Darme aus und zweitens auf dem Wege der Blutbahn mit Ausschcidung in die Galle. Die erste Annahme kann heute als verlassen gelten. War sie früher schon deshalb unwahrscheinlich, weil man bei Annahme der aszendierenden Infektion viel häufiger auch Gallenblasen, ohne daß ein Typhus bestand, durch andere Bakterien infiziert finden mußte, so mußte diese Meinung von dem Momente an fallen gelassen werden, als man die Kenntnis erlangte, daß der Typhus im Beginn keine Darmkrankheit, sondern eine Blutkrankheit ist. Die Infektion vom Blute aus wurde hierdurch wahrscheinlich und ist durch mehrfache Tierexperimente bewiesen worden. Zu erwähnen sind hier besonders die Tierversuche von $\left.\mathrm{D} \ddot{\mathrm{rrr}}{ }^{\mathbf{1}}\right), \mathrm{Koch}^{2}$ ) und $\mathrm{Ch}$ a $\mathrm{rolanza}^{3}$ ), zwischen denen allerdings, was den Weg der Blutinfektion anlangt, Widersprüche bestehen. Es können nämlich die Typhusbazillen mit der Galle ausgeschieden und dann vom Ductus hepaticus in die Gallenblase eingeschwemmt werden, oder aber die Typhusbazillen durchwandern die Kapillaren der Gallenblasenwand und gelangen auf diese Wiese in die Galle. Alle Versuche haben ergeben, daß im Gefolge von subkutanen Injektionen keine Typhusbazillen in der Gallenblase auftreten, wohl aber nach den Versuchen von $\mathrm{D}$ ör $\mathrm{r}$ nach intravenöser Injektion stets und nach den Versuchen von $\mathrm{C} h$ i a r o la $\mathrm{n} \mathrm{z}$ a in den meisten Fällen (nur in 26 Proz. konnte eine Infektion der Gallenblase nicht nachgewiesen werden). Der Weg der Infektion wurde von den Untersuchern durch Unterbindung des Ductus cysticus festzustellen versucht. 1)ie Versuche von Dör r haben hierbei ergeben, daß nach dieser Unterbindung keine Infektion der Gallenblase eintritt, während bei neun Versuchen, von $\mathrm{Koch}$ und $\mathrm{Ch}$ ia rola $\mathrm{nza}$ trotz Unterbindung des Ductus cysticus Typhusbazillen in der Gallenblase auftraten.

1) I) ör $\mathrm{r}$, Experimentelle Untersuchungen über das Fortwuchern von Typhusbazillen in der Gallenblase. Zentralbl. f. Bakt. Abt. I Orig. 1905, Bd. 39, S. 624.

2) Koch, Typhusbazillen und Callenblase. Zeitschr. f. Hygiene usw. I go8, 13d. 62, Heft I, S. I.

3) Chiarolanza, Beziehungen der Typhusbazillen zu der Gallenblase. Zeitschr. f. Hygiene usw. I908, Bd. 62, Ileft I, S. II. 
$\mathrm{D} \ddot{\mathrm{r}} \mathrm{r}$ schließt aus seinen Versuchen, daß die Infektion durch Ausscheidung der Bazillen mit der Galle in die Leber vor sich gehen mußte, während $\mathrm{Koch}$ und $\mathrm{Ch}$ i a rolanza ihre Versuche dahingehend verwerten, daß dieser Infektionsweg nicht möglich sei, vielmehr dic Infektion durch die Kapillaren in der Gallenblasenwand vor sich gehen müsse. Mit Recht weisen sie darauf hin, daB die negativen Resultate von $\mathrm{D}$ ö $\mathrm{r}$ vielleicht dadurch bedingt sind, da $B$ die Infektion überhaupt nicht in allen Fällen cintritt, da auch sie, ohne Unterbindung des Ductus cysticus, vereinzelt negative Resultate hatten. Diese beiden Autoren können ferner zur Begründung ihrer Ansicht auf ein Präparat einer Gallenblase hinweisen, das von einem in der dritten Woche des Typhus Gestorbenen herrührt, und bei dem man in den Gallenblasenpapillen um die GefäBe herum Bakterienhaufen sieht, die nach ihrer Annahme durch Vermehrung der Typhusbazillen nach Auswanderung aus den Gefäßen entstanden sind. Dieser Befund ist einzig dastehend und findet nicht allgemeine Anerkennung. So glaubt Frä nkel l), auf Grund sehr ausgedehnter an den verschiedensten Typhusorganen angestellter mikroskopischer Untersuchungen, daß die für Typhusbazillen charakteristischen Herdchen intravaskuläre, und zwar durch postmortale Vermehrung vital in die Blutbahn eingedrungener Typhuskeime entstandene Bazillenlager repräsentieren". Hierzu bemerke ich, da $B$ in den drei von mir mikroskopisch untersuchten, mit Typhusbazillen infizierten Gallenblasen es in keinem Falle gelungen ist, Typhusbazillenhaufen oder auch nur mit Sicherheit einzelne Typhusbazillen nachzuweisen, obwohl die Färbung mit Carbol-methylgrün-Pyronin nach den von $S$ a a th of ${ }^{2}$ ) angegebenen Vorschriften, die cine Gegenfärbung der Bazillen im Gewebe ergeben soll, vorgenommen wurde. Auch in dem von $\mathrm{Grimme}{ }^{3}$ ) beschricbenen Fall, bei dem ebenfalls in hiesiger Klinik die Cholc-

I) Fraenkel, Eugen, Über Typhus abdominalis und seine Bezichungen zu den Gallenwegen. Mitt. aus den Grenzgebieten der Chirurgie u. Medizin, I 909 , Bd. 20 , S. $898 \mathrm{f}$.

2) Sa a thoff, Die Nethylgrün-Pyronin Methode für elektive Färbung der Bakterien im Schnitt. Deutsche med. Wochenschr. I905, Nr. 51, S. 2047.

3) Gri m me, Über lyphusbazillenträger in den Irrenanstalten. Münchn. ned. Wochenschr. 1908. S. io. 
cystectomie bei einer Bazillenträgerin ausgeführt wurde, gelang es nicht, in Schnitten durch dic Gallenblasenwand Typhusbazillen nachzuweisen.

Den Kliniker interessieren besonders dic Folgen der Infektion der Gallenblase, die ihren Einfluß äußern kamn, erstens in der möglichen Bildung von Gallensteinen und zweitens in der dauernden Ansiedlung von Typhusbazillen in der Gallenblase.

Auf die erste Frage gehe ich hier nicht näher ein. Für einen gewissen ursächlichen Zusammenhang zwischen Cholelithiasis und Typhus sprechen aber jedenfalls verschiedene Punkte: In Gegenden, in denen Typhus herrscht, gibt es viele Gallensteinkranke, von beiden Krankheiten wird das weibliche Geschlecht und zwar in gleicher Weise bevorzugt (auf einen Mann kommen ca. 3-4 kranke Frauen) und nicht zuletzt zahlrciche Experimente, aus denen hervorgeht, dal $B$ war die verschiedensten Bakterienarten imstande sind, durch ihr Wachstum das in der Galle enthaltene Gemenge von gallensauren Silzen zu zersetzen, dab aber einigen Bakterienarten, so besonders denjenigen von der Typhusgruppe die Fähigkeit, die Galle zu zersetzen, in besonders hohem Grade zukommt. Hierdurch kann der Anstoß zur Gallensteinbildung gegeben werden, wie 1 . a. von $\mathrm{Ex}$ ner und $\mathrm{Heyrovsky}{ }^{1}$ ) nachgewiesen wurde. Andererseits kann man Lrsache und Wirkung umkehren, wie es $\mathrm{H}$ i s $\mathrm{ch}$ in dem erwähnten Vortrage tut, und sagen: Die Ursache der Infektion der Galle ist bedingt durch die vorher schon bestehende Stagnation der Galle. Entzündungserscheinungen und damit die Möglichkeit der dauernden Ansiedlung von Typhusbazillen werden also viel leichter bei Frauen, die aus anderen Gründen zur Gallensteinbildung neigen, eintreten.

Forster und Kayser') haben als erste auf die Bedeutung der Gallenblase bei den sogenannten Typhusbazillenträgern $^{3}$ ) hingewiesen, und zuerst die Gallenblase als Brutstätte

1) Exner und Heyrovsky, Die Pathogenese der Chololithiasis. Archiv f. klin. Chirurgie I908, 13d. 86, S. 6009.

2) Forster und $\mathrm{K}$ a y ser, Cber das Vorkommen von Typhusbazillen in der Galle von Typhuskranken und Typhusbazillenträgern. Münchn, med. Wochenschr. 1905, Nr. 31 .

3) Die Nomenklatur ist in den neuesten Arbeiten keine einheitliche. Inter Typhusbazillenträgern werden meist Ieute verstanden, dic Typhusbazillen bis 
der Typhusbazillen erkannt. Seitdem ist in noch höherem Maße wie früher die Aufmerksamkeit auf diese Bazillenträger gelenkt, so daB ein großer Teil der von $\mathrm{Robert} \mathrm{Koch}$ inaugurierten Typhusbekämpfung in dem Aufsuchen der Bazillenträger besteht, um die Infektionen durch diese zu verhüten. In größeren $\mathrm{Zu}$ sammenstellungen findet man angegeben, da $B$ ungefähr 2-6 Proz. der an Typhus crkrankten später zu Dauerausscheidern werden. Dic Infektiosität dieser ist über allen $Z$ weifel festgestellt, und große Epidemien haben auf solche Personen mit aller Wahrscheinlichkeit bezogen werden können. So konnte Rosenth a $1^{1}$ ) Io Proz. aller Typhuserkrankungen, die im Verlauf von Io Jahren in Göttingen vorgekommen waren, auf eine Typhusbazillenträgerin zurückführen.

Um die große Ansteckungsgefahr, die von diesen Personen ausgeht, zu beseitigen, hat man die verschiedensten internen Mittel versucht. Man hat Abführmittel gegeben, man hat versucht die Typhusbazillen durch Verfütternug von unschädlichen Bakteriengemengen zu verdrängen, man hat Immunisierung versucht, alles ohne Erfolg. Nach der Erkenntnis, daß die Typhusbazillen ihren Sitz in der Gallenblase haben, hat man nach dem Vorschlag von Forster Gallenpräparate gegeben, um durch Anregung der Gallensekretion die Typhusbazillen auszuschwemmen, cbenfalls ohne Erfolg. Neuerdings ist von Lief $m$ a $\mathrm{n}^{2}$ ) die Behandlung mit Yoghurt auf Grund von Versuchen an zwei Typhusbazillenträgern empfohlen worden, und es ist mit diesem Mittel gelungen, dic Ausscheidung von Typhusbazillen zum mindesten wesentlich zu beschränken. Einen Dauererfolg verspricht

zu drei Monaten ausscheiden, unter Daucrausscheidern solche, die Typhusbazillen noch über diese Zeit hinaus absondern. Oder aber es werden als Bazillenträger Leute bezeichnet, dic ohne klinische Erkrankung gezcigt zu haben, Typhusbazillen absondern und als Dauerausscheider solche, die nach der Rekonvaleszenz noch den betreffenden Erreger ausscheiden. Ich verwende den Namen Typhusbazillenträger, wie dies in allen früheren Arbeiten geschah, für Personen, die, ohne klinisch krank zu sein, Typhusbazillen absondern, cinerlei, cb sie überhaupt keinen nachweisbaren Typhus gehabt oder ihn überstanden haben.

1) Rosenthal. Bericht über die 'lätigkeit des bakteriologischen Lntersuchungsamtes zu Göttingen. Hygienische Rundschau ig()6, Nr. 18/19.

2) Liefmann, Beitrag zur Behandlung der Typhusbazillenträger. Münchn. med. Wochenschr. 1909, S. 509 . 
sich aber der Verfasser anscheinend selbst nicht und empfichlt es mehr zur prophylaktischen Anwendung. Ein zuverlässiges internes Mittel besitzen wir also nicht und werden es voraussichtlich auch nicht erhalten, da die Beeinflussung der in der durch entzündliche Erscheinungen meist gestauten Galle befindlichen Typhusbazillen aussichtslos erscheint.

Deshalb ist es verständlich, $\mathrm{da} B$ eine chirurgische Behandlung von dem Momente an versucht wurde, wo man die Gallenblase als Sitz und Brutstätte der Typhusbazillen erkannte. Als erster hat Dehle ${ }^{1}$ ) eine chronische Bazillenträgerin durch einen operativen Eingriff an der Gallenblase zu heilen versucht. Er war hierzu ermutigt, besonders durch die Feststellungen Blumenthals $\left.{ }^{2}\right)$, der bei 3 wegen Gallensteinen operierten Kranken (Cholecystostomie), bei denen Typhusbazillen in der Galle nachgewiesen wurden, nach der Operation keine Typhusbazillen im Stuhle feststellen konnte, nur bei einem Kranken, der in der Gallenblase Paratyphusbazillen Typ A enthielt, konnten wenige Tage nach der Operation einmal die gleichen Bazillen aus dem Stuhle gezüchtet werden, verschwanden dann aber, und waren bis I Jahr darnach nie mehr nachzuweisen. D e h l e r wählte ebenfalls die Cholecystostomie, und es wurden bei dem einen Kranken in den nächsten Monaten bei r66 Stuhluntersuchungen nur dreimal sehr spärlich Typhusbazillen gefunden. In dem zweiten Falle konnten nach der Operation unter 30 Stuhlproben noch zweimal Typhusbazillen, ebenfalls sehr spärlich, nachgewiesen werden. Aus der in die Gallenblase eingeführten Drainage entleerten sich reichlich die gleichen Keime. Die Nachbehandlung erfolgte mit Spülungen der Gallenblase. Auf Grund dieser Beobachtungen empfahl D e h l e r ein gleiches Verfahren für Bazillenträger, bei denen zwar im Stuhl, aber nicht im Blut und Urin Typhusbazillen nachweisbar sind. Er glaubte hierzu auf Grund der Erfolge der Operation berechtigt zu sein, da bei der ersten Bazillenträgerin vor der Operation unter 37 Untersuchungen

I) Dehler, Zur Behandlung der Typhusbazillenträger. Münchn. med. Wochenschr. 1907, Nr. 16 und 43 .

2) Fr. Blu menthal, lber das Vorkommen von Typhus und Paratyphusbazillen bei Erkrankungen der Callenwege. Münchn. med. Wochenschr. 1904, Nr. 37 und med. Ḱlinik 1905. Nr. $4^{8 .}$ 
jedesmal reichlich Typhusbazillen und bei der zweiten in über der Hälfte der Stuhlproben Typhusbazillen nachweisbar waren. IDenn es lag weiter die Möglichkeit vor, daß eine Infektion des Stuhles mit Typhusbazillen von einer anderen Trägerin, die auf demselben Saale lag, ausgegangen war. Daß in der Tat eine solche Infektion eintreten kann, konnte ich feststellen, da eine von Io unter Kontrolle des Cntersuchungsamtes ${ }^{1}$ ) in Göttingen stehenden Typhusbazillenträgern nur so lange die Bazillen ausschied, als sie Gelegenheit hatte, solche von einer andern Kranken, die auf demselben Saale lag, aufzunehmen. Seit der Mitteilung von De h le r haben sich eine Reihe Arbeiten mit der Möglichkeit der operativen Beseitigung der Typhusbazillen beschäftigt.

Fränckela) hat seine Ansicht dahin ausgesprochen, daß durch Eröffnung der Gallenblase und sorgfältige Ausspülung mit antiseptischen Flüssigkeiten eine Heilung möglich sci.

For ne ${ }^{3}$ ) teilte einen Fall von F orster mit: Eine Paratyphusträgerin, bei der wegen Gallensteinkoliken die Cholocystostomic ausgefülirt wurde, schied auch nach der Operation noch Paratyphusbazillen aus: For s t e r hält deshalb stets dic Ektomic für angezeigt.

L or e ${ }^{4}$ ) veröffentlichte einen Fall, bei dem wegen Cholecystitis die Ektomie ausgeführt wurde. Da der Mann 2 Jahre vorher einen Typhus überstanden hatte, wurde der Stuhl vor der Operation untersucht, und es wurden Paratyphusbazillen in ihm gefunden. Die Gallenblase enthielt die gleichen Bakterien. Nach der Operation aber konnten trotz mehrmaliger Untersuchung keine Paratyphusbazillen mehr im Stuhle nachgewiesen werden.

Evers und $\mathrm{Mühlens}{ }^{5}$ ) teilten einen Fall von Chole-

1) From me, A., Bericht über die Tätigkeit des bakteriologischen Untersuchungsamtes in Göttingen. Hygienische Rundschau 1907. Nr. 15.

2) Fracnkel. Zweite Tagung der freien Vereinigung für Mikrobiologic in Berlin Igo8, Zentralbl. f. Bakteriologie, 1. Abt. Referate, Bd. 42. Beiheft. S. $50 \mathrm{ff}$.

3) For ne t. Zentralbl. f. Bakteriologic. 1. Abt. Referate, Bd. 42, Beiheft.

4) Lorey, Uber einen Fall von Cholecystitis paratyphosa. Münchn. med. Wochenschr. I $908, \mathrm{Nr}$. I.

5) Evers und $M$ üh le $\mathrm{ns}$, Cholelithiasis paratyphosa und Paratyphuserkrankung, ein Beitrag zur Frage der Bazillenträger. Deutsch. Militärärztl. Zeitschr. I g09, Heft 9 . 
cystitis, bedingt durch den Paratyphusbazillus Typ B mit. Durch die Cholecystostomie wurde die Ausscheidung der Bazillen nicht beseitigt, sondern solche später häufig im Stuhle nachgewiesen, auch schon zu der Zeit, als sich die Galle aus der Fistel der Blase zum Teil entleerte und zahlreiche Bazillen mit ausschwemmte.

Die hiesige Heil- und Pflegeanstalt (Geh. Rat Cramer, veröffentlicht von $\mathrm{Gr}$ i $\mathrm{me} \mathrm{e}^{1}$ ), lieb eine Typhusträgerin in der hiesigen chirurgischen Klinik von Herrn Geheimrat $\mathrm{Br}$ a u n, der die Ektomie ausführte, operieren mit dem Resultate, da $B$ nur noch am II. und r5. Takge nach der Operation sich Typhusbazillen im Stuhle nachweisen ließen, seitdem aber keine mehr in den folgenden $7 \frac{1}{2}$ Wochen. Ich bemerke, daß ich diese Kranke jetzt noch zweimal nachuntersucht habe, und $\mathrm{da} B$ auch jetzt, also drei Jahre nach der Operation, keine Typhusbazillen nachgewiesen werden konnten. Die Publikation von $\mathrm{Gr}$ i m me findet sich in der neueren Literatur wohl deshalb so selten erwähnt, weil aus dem Titel nicht hervorgeht, daß die Heilung einer 'Typhusträgerin durch Operation dirin beschrieben wird.

Den Fall von Loele ${ }^{2}$ ) kann man für die Dauerheilung nicht verwerten, da die Patientin 4 Tage nach der Operation gestorben ist.

Die meisten Autoren versprechen sich von einer Operation keinen Erfolg und wollen die Operation auf die Fälle beschränkt wissen, bei denen eine Gallenblasenerkrankung eine Indikation abgibt.

F ränkel${ }^{3}$ ) erklärt cine Operation zur Bescitigung der Bazillenausscheidung direkt für zwecklos. Einen ähnlichen Standpunkt vertritt Loele. $\mathrm{H}$ a is ma ns ${ }^{4}$ ) glaubt sich von der Cholecystectomic keine besonders günstige Einwirkung versprechen zu können. P i c k ${ }^{5}$ ) sagt in einem Vortrag: „Typhus-

1) Grim me, l. c.

2) Loele, Typhusbazillenträger und Cholecystectomie. Deutsche med. Wochenschr. I909, Nr. 33.

3) Ir a enkel, l. c.

4) $\mathrm{H}$ u ismans, Ċor Typhusreinfektion, Münchn. med. Wochenschr. 1909, Nr. 38 .

5) Pick, liber die Genese der Urininfektion beim Abdominaltyphus: Verein für innere sledizin und Kinderheilkunde zu Berlin, Sitzung v. 4. VII. Io. Ref. Berl. Klin. Wochenschr, 1910, Nr. 3\%. 
bazillenträger sind nicht zu heilen, Cholektomie ist ohne Erfolg". Einen nicht ganz ablehnenden Standpunkt vertritt $\operatorname{Koch}^{1}$ ), da nach seiner Ansicht, mit der radikalen Entfernung der Gallenblase schon ein Hauptherd der Bazillen aus dem Körper entfernt wird". Forste ${ }^{2}$ ) empfichlt dic Ektomie, da er in dem schon erwähnten Falle durch die Stomie keine Heilung sah.

Schon nach der eben zusammengestellten Literatur scheint diese Operation mehr zu empfehlen $z u$ sein, denn ich finde zwar eine Reihe von Mißerfolgen bei der Stomic, aber auch nicht cine einzige bei der Ektomie.

Deshalb dürfte es von Interesse sein, wemn ich über das Heilungsresultat bei einigen Kranken berichte, bei denen in der Gallenblase Typhusbazillen nachgewiesen wurden. Alle wurden operiert, nicht weil man wußte, daß sic Typhusträger waren, sondern wegen Gallensteinerkrankung und zwar wurde bei allen die Cholecystectomie ausgeführt, die von Herrn Geheimrat B r a u n stets bevorzugt wird. Daß die Kranken Typhusbazillenträger waren, ist vorher nicht festgestellt worden. Ich nehme es aber als absolut sicher an, da der AbfluB aus der Gallenblase bei einer Kranken gar nicht behindert war, bei der zweiten fanden sich bei der Obduktion Typhusbazillen in der Leber und bei der dritten wurden Typhusbazillen noch eine kurze Zeit lang nach der Operation in den Fäces gefunden.

Die Krankenblätter lasse ich kurz folgen und schließe ihnen das Krankenblatt der Typhusträgerin, die im Jahre 1907 in hiesiger Klinik operiert wurde, an.

I. D. R., Postschaffnersfrau, 46 Jahre, aus E. Aufnahme 20. VIII. I909.

Anamnese: Vor 14 Jahren Typhus überstanden, zuerst erkrankte der Sohn, dann mit der Patientin selbst zu gleicher Zeit eine Tochter, seitdem gesund. Krankheitsfälle sollen in der Umgebung nicht vorgekommen sein. Seit 14 Tagen Gallensteinkoliken und geringe Gelbsucht. Status: ikterische Färbung der Skleren. Gallenblasengegend sehr druckempfindlich, hier undeutliche Resistenz zu fühlen. Stuhl gallig gefärbt. 23. VIII. Chloroformnarkose. Längsschnitt durch den Musculus rectus. Gallenblase ist prall gefüllt, mehrere Steine durchzufühlen. Nach Punktion Auslösung aus dem

I) $\mathrm{K} \circ \mathrm{ch}, \mathrm{I}, \mathrm{c}$.

2) Forster, 1. c. 
Lebcrbett, Unterbindung der Arteria cystica, Abbindung des Cysticus dicht am Choledochus, dieser ist frei von Steinen und nicht erweitert. Tampon, Bauchnaht. Aus der Galle, die fast klar und nur gering flockig ist, werden Typhusbazillen und Kokken (nicht näher identifiziert), gezüchtet ${ }^{1}$ ). lassen.

16. IX. nach glattem Verlauf mit kleiner Granulationsstelle ent-

29. IX. Vollständige Heilung.

Stuhluntersuchungen am 3. VIII., 6. VIII. und Io. VIII. I9Io negativ.

2. Ch. N., Steinbrechersfral, $4^{6}$ Jahre aus L. Aufnahme I. IX. I9lo.

A namnese: Vorher nie krank, nie typhusähnliche Krankheit durchgemacht, dagegen hat der Mann vor 5 Jahren Typhus überslanden. Seit $1-2$ Jahren vier heftige Schmerzanfälle in der rechten Oberbauchgegend, jetzt wieder seit 4 Wochen mit Gelbsucht.

Status: Kräftige Frau, kein Ikterus, Urin ohne Gallenfarbstoff, Stuhl gefärbt. In der Gallenblasengegend fühlt man cinen faustgroßen mit der Leber zusammenhängenden kugligen Tumor. Leib sonst weich und ohne Besonderheiten. 5. IX. 1909 Chloroformnarkose. Hakenschnitt, Gallenblase prall gefüllt, enthält dünnflüssigen Eiter und ist mit den Nachbarorganen so fest verwachsen, daßB sic scharf ausgelöst werden nub. Am Beginn des Cysticus ein kirschkerngroßer Verschlußstein, nach Punktion Ektomic. Choledochus ist frei von Steinen. Tampon, Bauchnant. Aus der Galle werden Typhusbazillen in Reínkultur gezüchtet. 7. IX. Heute plötzlich Herzschwäche, von der die Kranke sich nicht mehr erholt, 8. IX. Exitus. Io. IX. Sektion. Frische Pneumonie beider Unterlappen. Frische parenchymatöse Nephritis. Bei der Obduktion wurdc Stuhl aus dem Cöcum entnommen, in ihm wurden keine Typhusbazillen gefunden. Aus der Galle, die aus einem Schnitt durch die Leber aufgefangen wurde, konnten neben Bacterium coli und verschiedenen Fäulnisbakterien sehr zahlreich Typhusbazillen gezïchtet werden.

3. M. K., Landwirtsfrau, 45 Jahre, aus M. Aufnahme I4. II. I9og.

A namnese: Früher stets gesund, nie Typhus überstanden, auch von Typhuserkrankungen in der Umgebung nichts bekannt. Seit

1) Die Züchtung aus der Gralle erfolgte stets auf Drigalski-Conradi Agarplatten. Die weitere lintersuchung wurde durch Prüfung der Beweglichkeit, durch Wachstum in Bouillon, auf Gelatine und in Petruschkyscher I.akmusmolke vorgenommen. Die Stuhlzüchtungen wurden auf zwei Serien Drigalski-Conradi und Endo-Agarplatten ausgeführt. Die Sachprüfung aller reingezüchteten Stämme und ihre Agglutionation mit hochwertigem Serum wurde in freundlicher Weise im Untersuchungsamt des Hygienischen Instituts ausgeführt. 
November 1909 anfallsweise auftretende Schmerzen in der rechten Oberbauchgegend, die nach Rücken und Schulter ausstrahlten, seit November Gelbsucht, in der letzten Zeit alle 5-6 Tage ein Anfall. Status: Magere, leicht ikterische Frau, der Leib ist gespannt, die Gallenblasengegend sehr druckempfindlich, hier fühlt man am vorderen Rand der Leber einen etwa apfelgroßen Tumor. Stuhl acholisch. 15. II. Heute heftiger Kolikanfall mit starkem Ikterus. Morphium. I6. II. Immer noch heftige Schmerzen, Tumor ist größer. 4. III. Da der Ikterus nicht besser wird, heute in Chloroformnarkose Hakenschnitt. Gallenblase mit flüssiger Galle gefüllt, ohne Steine, dagegen sind mehrere große im prall gefüllten und erweiterten Choledochus zu fühlen. Choledochotomie. Entfernung der Stcine, es entleert sich reichlich grünliche Galle, Drainage des Choledochus. Cholecystektomic. Tamponade, Bauchnaht. Mikroskopische Präparate aus der Galle zeigen wenige Leukocyten und in enormen Haufen immer zu mehreren hundert zusammenhängend, wie agglutinierte Bakterien ausschende Stäbchen, keine deutliche Eigenbewegung. In Kulturen wachsen sehr reichlich Typhusbazillen ${ }^{1}$ ) in Reinkultur. Verlauf: Der Ikterus verschwand allmählich, Patientin konnte am 26. II. mit kleiner Fistel entlassen werden.

Aus der Choledochusdrainage konnten am 9. III. neben sehr zahlreichen Typhusbazillen ganz vereinzelte Kolonien von Bacterium coli und Bacillus pyocyaneus gezüchtet werden. 13. III. Drainage entfernt. Am 14. III, aus der Fistel abgeimpft: Fast nur pyocyaneus, ganz vercinzelt Typhusbazillen.

Stuhluntersuchungen. 17. III. Ganz vereinzelt Typhusbazillen. 24. III. Ein Fünftel der Kolonien sind Typhuskolonien. I. VIII. Die Kranke stellt sich in der Klinik vor, ist vollständig gesund. Stuhl ohne Typhusbazillen. Weitere Untersuchungen am 5. VIII., 7. IX. und 9. IX. waren negativ.

4. J. V., Arbeiterfrau, 38 Jahre, aus W. Aufnahme 17. VI. 1907.

Am 24. VI. 1907. Cholecystektomic, da Patientin Typhusbazillenträgerin ist (von Herrn Dr. Grim me ${ }^{2}$ ) veröffentlicht). Den Stuhl dieser früheren Trägerin, der von Iferrn Dr. Grimme bis 55 Tage nach der Operation bazillenfrei gefunden wurde, konnte am i8. VIII. I9IO und 22. VIII. I9IO nachuntersucht werden mit dem Resultate, daß auch jetzt keine Typhusbazillen gefunden wurden.

Von den drei neu veröffentlichten Kranken hat nur eine einen sicheren Typhus vorher überstanden, bei der zweiten hat der Ehemann 5 Jahre vorher Typhus gehabt, während bci der

1) Für die Identifizierung dieses Stammes, der im Anfang sehr schwer eigenbeweglich war, bin ich Herrn Dr. W. Rosenthal zu Dank verpflichtet.

2) Grim me, l. c. 
letzten von einer Typhuscrkrankung überhaupt nichts nachgewiesen werden konnte.

Zusammengefaßt ist also bei 3 Typhusbazillenträgcrinnen durch die Cholecystectomie Heilung erziclt worden. Besonderes Interesse bicten die zweite und dritte Kranke. Bei der zweiten konnten trotz Exstirpation der Gallenblase bei der 5 Tage nach der Operation vorgenommenen Obduktion in der Leber Typhusbazillen nachgewiesen werden, ein Zeichen dafür, daß nicht nur die Gallenblase, sondern auch die Gallengänge in der Leber infiziert waren. Noch besser konnte dieser Beweis bei der dritten Kranken erbracht werden. Bei dieser sind trotz Exstirpation der infizierten Gallenblase aus der Choledochusdrainage Typhusbazillen fast in Reinkultur abgesondert worden, um später von anderen Bakterien, namentlich dem Bazillus pyocyaneus, verdrängt zu werden. Aber selbst nach Weglassen der Drainage konnten aus der Fistel noch vereinzelt Typhusbazillen gezüchtet werden. Einen gleichen Fall habe ich in der Literatur nicht verzcichnet gefunden, denn der Fall von $\mathrm{L}_{\text {o e }} \mathrm{e}^{\mathbf{1}}$ ) war durch einen gleichzeitig neben der Gallenblase vorgefundenen AbszeB und durch eine Verletzung des Duodenums, die den Tod am 4. Tag veranlaßte, kompliziert. Der einwandfreie Beweis ist jedenfalls damit am Lebenden erbracht, daß nicht nur die Gallenblase, sondern auch die Gallengänge einen Aufenthaltsort für die Typhusbazillen darstellen. Der Befund bei Sektionen hatte dieses allerdings schon fast mit Sicherheit annehmen lassen.

Auf Anregung von Herrn Geheimrat $\mathrm{B} r$ a $\mathrm{u} n$ habe ich in den letzten 2 Jahren nicht nur stets eine bakteriologische Untersuchung des Gallenblaseninhaltes vorgenommen, sondern auch die durch die Choledochusdrainage ausgeschwemmte Galle untersucht. Hierbei hat sich ergeben, daß bei Stauung im Choledochus fast stets eine Infektion der Gallenblase und der Gallenwege, meist mit Bacterium coli, eingetreten ist, und $\mathrm{da} B$ die Choledochusdrainage bei infizicrter Gallenblase stets die gleichen Bakterien und zwar in sehr reichlichem Maße entleert, wie sie in der Gallenblase gefunden wurden.

I) $\mathrm{L}, \mathrm{e} \mathrm{le}, \mathrm{l} . \mathrm{c}$. 
Trotzdem wird in allen diesen Fällen, wenn keine sonstigen Komplikationen eintreten, Heilung crzielt, und es ist nicht bekannt, da $B$ dieser Bakteriengehalt der Leber nach der Operation einen schädlichen Einfluß auf den Kranken ausgeübt hätte, wenn nur die Drainage gut funktioniert. Deshalb ist als sicher anzunehmen, daB, sobald der ungestörte Abfluß der Galle durch die Operation erreicht ist, durch Ausschwemmung der Bakterien die Galle in den meisten Fällen wieder steril wird.

Bei der Infektion mit Typhusbazillen muß es gerade so sein. Es muß aber in diesen Fällen die Gallenblase radikal entfernt werden, da die Galle einen sehr guten Nährboden für die Typhusbazillen abgibt; denn nur dann fällt der Schlupfwinkel, in dem immer von neuem eine Vermehrung der Typhusbazillen eintreten kann, fort, und es scheint trotz gleichzeitiger Infektion der höheren Gallenwege zur vollkommenen Heilung zu kommen. Aber auch spontane Heilungen bei Typhusbazillenträgern sind beschricben, so von Forster ${ }^{1}$ ), der eine solche durch den Eintritt eines Gallensteinanfalles, der sehr bemerkenswerterweise der erste hei der Kranken war, eintreten sah. Er nimmt an, daB in solchen Fällen ,mit der Aufhebung von Stauungen und der Ausspülung der Residualgalle durch die stetig sezernierte frische Galle die Typhusbazillen ohne weitere Vermehrung aus der Gallenblise entfernt werden können.

Ob durch die Entfernung der Gallenblase mit Sicherheit auf eine Heilung zu rechnen ist, läßt sich auf Grund der wenigen bisher beobachteten Fälle nicht sagen. Ich habe aber diese Beobachtungen zusammengestellt, um eine Nachprüfung von chirurgischer Seite zu veranlassen. Denn der Chirurg operiert sicher viel häufiger, als bekannt ist, Bazillenträger, bei denen ein Gallensteinleiden klinische Erscheinungen macht, die aber selbst keine Ahnung von der Infektiosität für ihre Umgebung haben. Durch Nachuntersuchungen dieser Kranken wird dann ein genügendes Material gesammelt werden können, um auch darüber ein Lrteil zu erhalten, ob man einem klinisch nichtkranken Bazillenträger eine Heilung durch Operation, die dann stets in der

I) Forster, Über die Beziehungen des Typhus u. Paratyphus zu den Gallenwegen, Münchn. med. Wochenschr. 1908, Nr. r. 
Cholecystectomie bestehen müßte, in Aussicht stellen kann. Denn unter der immer größer werdenden Menge der entdeckten Bazillenträger wird es häufig Leute geben, dic dic Gefahr, in die sie ihre nächste Lmgebung bringen, richtig erkennen, und dic mit Rücksicht auf ihre Angehörigen, die natürlich am leichtesten einer Infektion ausgesetzt sind, auf Beseitigung dieser Ansteckungsgefahr drängen und sogar selbst bereit sein werden, sich einer Operation zu unterziehen, wenn durch diese eine sichere Heilung in Aussicht gestellt werden kann.

Auch die Gefahren, in die die Bazillenträger durch ihre cigenen Bazillen kommen, scheinen größer zu sein, als man bisher annahm. Denn in neuester Zeit sind eine ganze Reihe von sogenannten Autoinfektionen, die sogar zum Tode geführt haben, bekannt geworden (Levy und Kayser ${ }^{1}$ ), Grimme ${ }^{2}$ ), $\mathrm{K}$ a $\left.\mathrm{m} \mathrm{m}^{3}\right)$ ). Diese Autoinfektionen kommen wahrscheinlich nicht so selten vor, denn die meisten Bazillenträger haben keine Ahnung von ihrem Leiden. Ohne diese vorherige Kenntnis aber begegnet die Diagnose einer Typhussepsis stets noch großen Schwierigkeiten, und sie kann nur durch besondere klinische Untersuchungsmethoden sichergestellt werden.

I) Levy und Kayser, Bahteriologischer Befund bei der Autopsie cines 'Typhusbazillenträgers. Hünchn. med. Wochenschr. I906, Nr. 50.

2) Grim me, Ein unter dem Bilde der Weilschen Krankheit verlaufender Fall von Typhus abdominalis, entstanden durch Autoinfektion von der Gallenblase her. Münclun. med. Wochenschr. 1907, Nr. 37.

3) $\mathrm{Ka} \mathrm{m} \mathrm{m}$, Die Gefährdung des Iyphusbazillenträgers durch dic eigenen Typhusbazillen. Münchn. med. Wochenschr. 1909. Nr. 20. 